\title{
Análisis Temático de los Tuits de los Líderes Políticos Españoles en las Elecciones Generales de 2016
}

\author{
Ricardo ZUGASTI, PhD \\ Departamento de Lingüística General e Hispánica \\ Facultad de Filosofía y Letras \\ Universidad de Zaragoza, Spain \\ E-mail: rzugasti@unizar.es
}

\section{An Issues-Based Analysis of the Tweets Published by the Spanish Political Leaders during 2016 General Elections}

\begin{abstract}
This article focuses on the issues published in Twitter by the main four Spanish political leaders during the general elections held on 26 June 2016. The research method is a quantitative content analysis applied to the each and every 2,339 tweets published by Mariano Rajoy (PP), Pedro Sánchez (PSOE), Pablo Iglesias (Podemos) and Albert Rivera (Ciudadanos) during the official electoral campaign. The objectives are to identify the campaign issues and to compare their presence between leaders. The results show that the main issue in both elections is the own party campaign activities and that there are manifest diferences between the four candidates. Regarding the comparison between shared issues, Rajoy and Rivera highlight the economic issues; Sánchez and Iglesias underline the gender-related demands; Rivera, Sánchez and Iglesias attach importance to the social rights; while Iglesias and Rivera focused on corruption and political regeneration.
\end{abstract}

Keywords: Spain; Electoral Campaign; Twitter; Campaign Issues; Political Leaders. 


\section{Introducción}

Las elecciones generales del 26 de junio de 2016 fueron una consecuencia del fin del bipartidismo en el Congreso de los Diputados, principal cámara parlamentaria española, que elige al presidente del gobierno de entre sus miembros. Las anteriores elecciones generales, celebradas el 20 de diciembre de 2015, inauguraron un nuevo escenario parlamentario, pues supusieron la fuerte irrupción en el Congreso de dos nuevos partidos políticos: Podemos, que alcanzó 69 escaños, y Ciudadanos, que obtuvo 42. Los dos grandes partidos hasta entonces, Partido Popular y Partido Socialista, se quedaron en 123 y 90, respectivamente.

La fragmentación del Congreso en cuatro grandes fuerzas y la ausencia de pactos factibles entre ellas hicieron inviable el nombramiento de un presidente del gobierno, lo que provocó la disolución de las Cortes y la convocatoria de unas nuevas elecciones para el 26 de junio de 2016, una situación inédita en el actual periodo democrático.

Los nuevos comicios confirmaron el final del bipartidismo y la composición del Congreso en cuatro grandes grupos parlamentarios. En esta ocasión el Partido Popular obtuvo 137 diputados; el Partido Socialista Obrero Español, 85; Unidos Podemos (Podemos en coalición con Izquierda Unida), 71; y Ciudadanos, 32. No obstante, tras estas elecciones, Mariano Rajoy, candidato del PP, pudo ser investido presidente gracias fundamentalmente al apoyo de Ciudadanos y a la abstención de buena parte de los diputados del PSOE.

El origen de Ciudadanos y de Podemos, las dos fuerzas que han quebrado el bipartidismo, se produjo en un caldo de cultivo común basado, de manera general, en las duras consecuencias de la crisis económica que comenzó en 2009 y en el desgaste acumulado de la legitimidad política de las instituciones españolas, incluida la Corona, y de la clase política. Buena parte de su éxito se explica por su capacidad para capitalizar ese descontento social hacia los viejos partidos de un sistema visto como necesitado de regeneración.

Pese a su diferente ubicación ideológica -en el centro liberal Ciudadanos y a la izquierda de la socialdemocracia Podemos- ambas formaciones presentan rasgos comunes como el hiperliderazgo de Iglesias y Rivera (Ellakuría y De Paco 2015) y el uso estratégico de los medios de comunicación y de las redes sociales para poder culminar su proyección a nivel nacional (López-García 2016).

La novedad que suponía en España la repetición de unas elecciones generales y la presencia en el terreno de juego político de dos partidos nuevos y fuertes que además concedían importancia a las redes sociales en su estrategia de comunicación política eran elementos que justificaban la pertinencia de un trabajo como el presente.

Este artículo se ubica, por lo tanto, entre las investigaciones que abordan el análisis de campañas electorales en Twitter, y acomete las últimas elecciones gene- 
rales celebradas en España, las de 2016. El trabajo se centra en comparar los temas (los issues de campaña, por utilizar en extendido término en inglés) publicados por cada uno de los líderes de los cuatro principales partidos nacionales en sus cuentas personales de Twitter. Se pretende, por lo tanto, un objetivo general consistente en conocer los temas de los tuits publicados por Mariano Rajoy (PP), Pedro Sánchez (PSOE), Pablo Iglesias (Podemos) y Albert Rivera (Ciudadanos) durante los quince días de duración oficial de la campaña electoral y durante la jornada de reflexión. En relación con lo anterior, los objetivos concretos de la investigación son, en primer lugar, identificar los temas presentes en cada una de las cuatro cuentas de Twitter y, en segundo lugar, comparar las cuentas de Twitter de los líderes atendiendo a los temas presentes.

\section{Marco teórico}

Las redes sociales se han incorporado al arsenal comunicativo empleado por los partidos políticos y, en concreto, por los candidatos durante una campaña electoral, periodo en el que los mencionados actores son más activos en estos medios sociales (Abejón-Mendoza, Sastre-Asensio y Linares-Rodríguez 2012). Pese a ello, las redes sociales no han sustituido a los medios convencionales, sino que se suman a las estrategias híbridas de comunicación de partidos y candidatos (Casero-Ripollés, Feenstra y Tormey 2016). Desde la perspectiva de las audiencias, éstas pueden mezclar la información tomada de unos y de otros medios, en un proceso conceptualizado como transmediación (Cheong y Lundry 2012). Además, las redes sociales funcionan como cajas de resonancia de la cobertura periodística tradicional de los actos de campaña (Lilleker, Darrer y Jackson 2010). En relación con lo anterior, por ejemplo, destaca el hecho de que la mayoría de los usuarios jóvenes acceden a los contenidos de la prensa digital gracias a los enlaces que encuentran en las redes sociales (Santín y Álvarez-Monzoncillo 2017).

Pese a la hibridación en las estrategias comunicativas, lo más habitual es que candidatos y partidos usen los nuevos medios sociales para difundir sus propios mensajes sin pasar por la mediación de los órganos periodísticos (Cammaerts 2012) e intentar así una influencia directa en las percepciones y conducta de los votantes (Zamora-Medina, Sánchez-Cobarro y Martínez-Martínez 2017). Los actores políticos no aprovechan, por lo tanto, la capacidad de interacción con los ciudadanos que les proporcionan las redes sociales, pues en campaña electoral prefieren un mayor control sobre sus mensajes (Alonso-Muñoz, Miquel-Segarra y Casero-Ripollés 2016).

De entre las diferentes redes sociales, Twitter se considera desde su popularización la más destacada en el ámbito político en el contexto español (Rodríguez y Ureña 2012), una preferencia que tiene que ver con su carácter de red abierta o pública, en la que no es necesario estar registrado o tener un perfil para acceder 
a la información, frente a otras más restrictivas como Facebook (Jivkova-Semova, Requeijo-Rey y Padilla-Cas 2017).

Algunas características de los usuarios españoles de Twitter contribuyen a acrecentar el interés de los actores políticos por esta plataforma de comunicación. En 2015, el 78\% de los tuiteros españoles tenía menos de 45 años, el 41\% había cursado estudios universitarios y el $20 \%$ gozaba de ingresos económicos que les ubicaba entre el $25 \%$ de la población con ingresos más altos. Además, los usuarios españoles de Twitter señalan que están fuertemente interesados en política mucho más que los usuarios de Internet en general (Global Web Index 2015).

Las investigaciones académicas sobre Twitter y política aplicadas al contexto español se han centrado mayoritariamente en cómo lo usan los partidos y los candidatos en campaña electoral. Asimismo, suelen enfocarse total o parcialmente en analizar los temas de campaña que se difunden a través de esta plataforma de comunicación. Considerando únicamente las elecciones generales, los trabajos que analizan los temas difundidos por los actores políticos en Twitter comenzaron con los comicios de 2011 (Lafuente y Verón 2013; Zamora y Zurutuza 2014; García y Zugasti 2014) y continuaron con el análisis de las generales de 2015 (López-García 2016; Pallarés y García 2016; Zugasti y García 2018). Este artículo sigue esta estela, si bien aporta la novedad de abordar las últimas elecciones generales celebradas en España, las de 2016.

\section{Metodología}

El método de investigación empleado fue el análisis de contenido cuantitativo, cuyas principales características son las siguientes: "Objetividad, sistematicidad y cuantificación de los contenidos manifiestos de la comunicación" (Cea 2001, 351). Es la técnica más adecuada para analizar un considerable volumen de contenidos textuales, como sucede en este trabajo, con una muestra compuesta por 2.339 tuits y retuits publicados en las cuentas de los líderes de los cuatro principales partidos políticos con dimensión nacional: Mariano Rajoy (@marianorajoy), Pedro Sánchez (@sanchezcastejon), Pablo Iglesias (@Pablo_Iglesias_) y Albert Rivera (@Albert_Rivera).

En la tabla I se concreta el número de tuits por candidato, así como el porcentaje que suponen del total de los mensajes publicados por los cuatro. En ella se percibe que tanto Rajoy como Sánchez y Rivera publicaron un número de tuits que puede calificarse como similar, incluso asumiendo que Sánchez publicó unos cien menos. La principal diferencia estriba en Pablo Iglesias, quien lanzó únicamente 124 mensajes durante la campaña electoral, un dato que debe ser tenido en cuenta a la hora de interpretar los resultados. El motivo de su menor frecuencia de publicación obedece a que incluyó menos retuits que sus rivales. 
TABLA I. Tuits publicados por cada candidato

\begin{tabular}{lcc}
\hline \multicolumn{1}{c}{ Candidato } & Número & Porcentaje \\
\hline Mariano Rajoy & 784 & $33,5 \%$ \\
\hline Pedro Sánchez & 673 & $28,8 \%$ \\
Pablo Iglesias & 124 & $5,3 \%$ \\
\hline Albert Rivera & 758 & $32,4 \%$ \\
\hline \multicolumn{1}{c}{ Total } & 2.339 & $100 \%$ \\
\hline
\end{tabular}

El análisis de contenido empleó un código de elaboración propia diseñado para dar respuesta a las preguntas de investigación del trabajo. Este código se aplicó de manera exhaustiva a cada uno de los tuits publicados durante los quince días de duración de la campaña electoral oficial y durante la jornada de reflexión. Para la obtención de las frecuencias que constan en las tablas incluidas en el artículo, los datos fueron procesados estadísticamente mediante el programa informático SPSS.

Las preguntas de investigación fueron las siguientes:

PI1: ¿Cuáles fueron los principales temas de campaña en cada una de las cuentas analizadas?

PI2: ¿Cuál fue la importancia de cada uno de los temas para cada candidato?

PI3: ¿Cuáles fueron las diferencias o similitudes entre los líderes políticos según los temas?

De las preguntas de investigación 1 y 2 deriva la primera hipótesis (H1): los cuatro candidatos analizados utilizaron Twitter mayoritariamente para difundir sus respectivos actos y eventos de campaña, haciendo gala en este sentido de un uso autorreferencial. La hipótesis se fundamenta en que este empleo ha sido demostrado por recientes investigaciones como las de Zugasti y Sabés (2015), Zugasti y Pérez González (2016), Pallarés y García (2017), o Zugasti y García (2018).

De la PI3 emerge la segunda hipótesis (H2): en el resto de temas, dejando a un lado los actos de campaña, existirán diferencias entre la importancia concedida a unos u otros asuntos por parte de los diferentes líderes. Dicha hipótesis se sustenta en trabajos que han demostrado estas diferencias temáticas entre candidatos en anteriores elecciones (García y Zugasti, 2016; Zugasti y García, 2018). Algunas diferencias eran palpables incluso al margen de ideologías políticas, entre los candidatos de los partidos emergentes frente a los de los partidos tradicionales.

\section{Resultados y Discusión}

Como muestra la tabla II, el presidente del gobierno y candidato del PP en las elecciones dedicó la mayor parte de sus tuits (36\%) a difundir y promocionar las propias actividades y eventos de campaña, un empleo de Twitter habitual en cam- 
paña electoral y que, como se verá, fue común a sus rivales políticos. La economía fue, por otro lado, un asunto muy presente en la cuenta de Mariano Rajoy, con un 19,1\% de los tuits centrados en ella. La mejora de los indicadores económicos en España fue utilizada por Rajoy como uno de los puntos fuertes de su gestión como gobernante, lo que se percibe en la elevada frecuencia de emisión de tuits con dicho asunto.

Los mensajes que aludían al populismo o a los radicalismos políticos registraron una notable presencia en el Twitter del candidato del PP: un 6,5\% de sus tuits durante la campaña hablaron de ello. Cabe destacar que la mayoría de estos tuits hacían referencia directa o indirecta a Podemos o a alguno de sus líderes, considerados populistas y radicales de izquierda por Rajoy.

Tras los tres grandes temas para el candidato del PP, el análisis presenta dos más con una presencia destacada y similar. La organización territorial de España aglutinó el 4,9\% de los tuits. En relación con este asunto, destaca el peso que tuvieron los mensajes en torno al proceso independentista de Cataluña, tema destacado para el líder conservador. Por otro lado, los pactos postelectorales entre diferentes partidos acumularon el 4,5\% de los tuits. En un escenario electoral complejo tras la fuerte irrupción de fuerzas políticas nuevas y tras la fallida experiencia de las elecciones de diciembre de 2015, que dieron lugar a un parlamento dividido e incapaz de investir a un nuevo presidente del gobierno, las alianzas entre formaciones políticas se conviertiron en un asunto crucial para quien finalmente consiguió renovar su mandato como presidente.

El único tema de índole internacional que tuvo un peso específico en el Twitter de Rajoy fue el conocido como Brexit, con el 2,8\% de los tuits. La salida del Reino Unido de la Unión Europea fue, como se verá, el único asunto extranacional -si bien con poderosas vinculaciones nacionales- tratado durante la campaña por los cuatro líderes en sus cuentas.

Los derechos y los servicios sociales presentaron una presencia discreta, con un $2,3 \%$. Los recortes a servicios públicos como, por ejemplo, la educación y la sanidad en el contexto de la grave crisis económica fueron una de las acusaciones del resto de formaciones políticas al gobierno liderado por Rajoy. No es extraño, por lo tanto, que en su cuenta intentara no incidir en un tema que le era conflictivo. Algo similar, aunque en mayor medida, sucedió con la corrupción política y la consiguiente necesidad de regeneración. Buena parte de los escándalos afectaban al partido gobernante, lo que propició que este asunto tuviera una escasa presencia en el Twitter de su candidato: únicamente el 1,5\% de los tuits hablaron de ello. Los debates electorales fueron el último asunto con entidad propia en la cuenta de Rajoy, con un escueto 1,3\%. 
TABLA II. Temas principales de los tuits de Mariano Rajoy (PP)

\begin{tabular}{lc}
\hline \multicolumn{1}{c}{ Temas } & $\%$ \\
\hline Organización/actividades de campaña & 36 \\
\hline Economía & 19,1 \\
\hline Populismos/radicalismos & 6,5 \\
\hline Organización territorial del Estado & 4,9 \\
\hline Pactos entre partidos políticos & 4,5 \\
\hline Brexit & 2,8 \\
\hline Derechos/servicios sociales & 2,3 \\
\hline Corrupción/necesidad de regeneración & 1,5 \\
\hline Debates electorales & 1,3 \\
\hline Reivindicaciones feministas/de género & 0 \\
\hline Otros $\quad$ Total & 21,1 \\
\hline
\end{tabular}

Como recoge la tabla III, el candidato del Partido Socialista a la presidencia del gobierno dedicó el 40,1\% de sus tuits a los actos de campaña de su propio partido. Otra vez se demuestra el predominio constante de este tema en las campañas electorales en Twitter.

Aparecen a continuación tres asuntos con una frecuencia similar, en torno al $8 \%$, que constituyen de facto los más destacados de la cuenta de Pedro Sánchez. Como se ha explicado al hablar de los temas de Rajoy, los casos de corrupción política afectaban principalmente al Partido Popular, por lo que eran utilizados por el líder socialista para lanzar mensajes que erosionaran a su principal rival, de ahí que el 8,8\% de los tuits versaran sobre ello. Prácticamente el mismo relieve tuvieron los derechos y prestaciones sociales $(8,7 \%)$, un asunto igualmente empleado en numerosas ocasiones por Sánchez para acusar al líder popular de ser el causante de los recortes y del deterioro del sistema público de bienestar. Los obligados pactos entre fuerzas políticas tras la celebración de las elecciones fueron el objeto del 8,3\% de los mensajes publicados por Pedro Sánchez. Una vez más, fue un asunto que cobró relieve dada la peculiar situación política española tras la irrupción de nuevas formaciones políticas y tras las fallidas elecciones de diciembre de 2015.

La economía tuvo una presencia moderada con un 6,5\% de los tuits, un dato que contrasta con la importancia que tuvo en la cuenta del candidato del Partido Popular. Por otro lado, los mensajes de apoyo a las reivindicaciones feministas o de género presentaron en el Twitter de Sánchez una frecuencia digna de mención $(3,3 \%)$ si se tiene en cuenta que no fue uno de los grandes temas que articularon la campaña y que, por ejemplo, no mereció tuit alguno por parte de su rival Rajoy.

Con un índice de aparición similar entre sí figuran los populismos y radicalismos políticos $(2,2 \%)$, que fueron en su mayor parte acusaciones de ser tal a 
Podemos y a sus líderes, y el Brexit (2,1\%), de manera parecida a su inclusión entre los temas de Rajoy. La organización territorial de España es el último tema en figurar con entidad propia en la cuenta del líder socialista con un 1,2\% de sus tuits. Los debates electorales no son, por último, merecedores de ningún tuit para Pedro Sánchez.

TABLA III. Temas principales de los tuits de Pedro Sánchez (PSOE)

\begin{tabular}{lc}
\hline \multicolumn{1}{c}{ Temas } & $\%$ \\
\hline Organización/actividades de campaña & 40,1 \\
\hline Corrupción/necesidad de regeneración & 8,8 \\
\hline Derechos/servicios sociales & 8,7 \\
\hline Pactos entre partidos políticos & 8,3 \\
Economía & 6,5 \\
\hline Reivindicaciones feministas/de género & 3,3 \\
Populismos/radicalismos & 2,2 \\
\hline Brexit & 2,1 \\
Organización territorial del Estado & 1,2 \\
\hline Debates electorales & 0 \\
\hline Otros $\quad$ Total & 18,8 \\
\hline
\end{tabular}

La tabla IV ofrece los resultados relativos a Pablo Iglesias, el líder del nuevo partido de izquierda Podemos, que se convirtió en la tercera fuerza política en el Congreso de los Diputados tras las elecciones de diciembre de 2015. Una vez más, el tema estrella para el líder de la formación morada fue la difusión de los diversos actos y eventos de su partido: un $46 \%$ de sus tuits se centraron en ello.

Destaca la importancia del segundo asunto más presente en la cuenta de Iglesias, la corrupción política y la necesidad de regeneración, que aglutinó el 10,5\% de sus tuits. Era éste uno de los asuntos primordiales del relato político que Podemos ha ofrecido desde su nacimiento como partido y que sigue siendo, como muestran los datos del análisis, uno de los pilares comunicativos de su líder. Esto es, el de un partido que nació para regenerar el sistema político español, ocupado y dominado por una casta de políticos que, en connivencia con determinados poderes económicos, han gobernado buscando su beneficio y enriquecimiento particulares.

Temas con notable y pareja presencia en la cuenta de Iglesias fueron las reivindicaciones feministas y de género (4,8\%). En la estrategia comunicativa en Twitter del candidato de Unidos Podemos a la presidencia del gobierno se percibe, por ello, un intento por constituir el referente político de estas demandas. Un $4 \%$ de los tuits de Iglesias tuvieron como tema los derechos y prestaciones sociales. En línea similar a lo comentado con Pedro Sánchez, eran mayoritariamente mensa- 
jes que responsabilizaban al gobierno del Partido Popular de los recortes en estos ámbitos.

El Brexit fue también en el caso de Pablo Iglesias el único asunto internacional con presencia de relieve en su Twitter $(2,4 \%)$, con una frecuencia de aparición similar además a la de los candidatos ya analizados. Los debates electorales y la economía fueron dos temas con la misma presencia en la cuenta del líder de Podemos: 1,6\%. Destaca el poco relieve de la economía entre los temas de campaña difundidos por Iglesias.

Por último, hay tres temas sobre los que no se publicó ningún tuit en @Pablo_Iglesias_: la organización territorial de España, los pactos entre los partidos, y los populismos o radicalismos políticos. Se vislumbra, por lo tanto, una intención por parte del candidato de Unidos Podemos de obviar determinados asuntos que podían serle perjudiciales en su estrategia comunicativa.

TABLA IV. Temas principales de los tuits de Pablo Iglesias (Podemos)

\begin{tabular}{lc}
\hline \multicolumn{1}{c}{ Temas } & $\%$ \\
\hline Organización/actividades de campaña & 46 \\
\hline Corrupción/necesidad de regeneración & 10,5 \\
\hline Reivindicaciones feministas/de género & 4,8 \\
\hline Derechos/servicios sociales & 4 \\
\hline Brexit & 2,4 \\
\hline Debates electorales & 1,6 \\
\hline Economía & 1,6 \\
\hline Populismos/radicalismos & 0 \\
\hline Pactos entre partidos políticos & 0 \\
\hline Organización territorial del Estado & 0 \\
\hline Otros Total & 29,1 \\
\hline \multicolumn{1}{c}{$\quad 100$} \\
\hline
\end{tabular}

Albert Rivera fue, como muestra la tabla $\mathrm{V}$, el líder político que hizo un menor uso de Twitter como tablón de anuncios de los actos de campaña. Si bien éste fue el tema más presente en su cuenta $(18,2 \%)$, está muy lejos del índice de aparición registrado en las respectivas cuentas de sus oponentes políticos.

La consecuencia de lo anterior fue que el líder de Ciudadanos ofreció más importancia a una mayor pluralidad de asuntos. Así, destaca la dimensión otorgada a la corrupción política y a la necesidad de regeneración del sistema democrático. De manera similar al discurso de Podemos, Ciudadanos también esgrimió el mensaje de ser un partido de nuevo cuño, alejado de los dos grandes partidos del sistema bipartidista anterior, PP y PSOE, y de los habituales escándalos de corrupción que afectan a estos. 
Destaca asimismo el peso de los pactos entre partidos en los mensajes emitidos por Albert Rivera $(10,4 \%)$, motivada primordialmente por saberse la fuerza política que, por su carácter centrista, podría garantizar la gobernabilidad del país a través de su apoyo tanto al PP como al PSOE. La economía tuvo igualmente una importancia considerable para Rivera: un 9,7\% de sus mensajes la tuvieran como tema, un dato que refleja el peso de la economía en el discurso político del líder catalán.

Los derechos y los servicios sociales aglutinaron el 5,6\% de los tuits de Rivera, un dato que otorga cierta relevancia a este tema, al igual que sucede con la organización territorial del Estado, un asunto que concentró el 3,8\% de los mensajes. Buena parte de estos tuits fueron críticas a los afanes independentistas del nacionalismo catalán, asunto especialmente sensible para Ciudadanos, un partido que nace en Cataluña con un nítido enfoque antinacionalista.

Parecida presencia entre sí tuvieron los populismos/radicalismos y los debates electorales: $2,9 \%$ y $2,8 \%$, respectivamente. Los tuits sobre populismo se centraron normalmente en ataques a Podemos, como sucedió en el caso de los mensajes de otros candidatos. La salida de Reino Unido de la Unión Europea fue, como para el resto de líderes, el único tema internacional con entidad propia $(1,1 \%)$.

Por último, Albert Rivera fue, junto a Rajoy, el único de los cuatro candidatos que no publicó tuits sobre las reivindicaciones de género.

TABLA V. Temas principales de los tuits de Albert Rivera (Ciudadanos)

\begin{tabular}{|c|c|}
\hline Temas & $\%$ \\
\hline Organización/actividades de campaña & 18,2 \\
\hline Corrupción/necesidad de regeneración & 17,1 \\
\hline Pactos entre partidos políticos & 10,4 \\
\hline Economía & 9,7 \\
\hline Derechos/servicios sociales & 5,6 \\
\hline Organización territorial del Estado & 3,8 \\
\hline Populismos/radicalismos & 2,9 \\
\hline Debates electorales & 2,8 \\
\hline Brexit & 1,1 \\
\hline Reivindicaciones feministas/de género & 0 \\
\hline Otros & 28,4 \\
\hline Total & 100 \\
\hline
\end{tabular}

\section{Conclusiones}

La primera hipótesis planteada en este trabajo queda confirmada a la luz de los resultados: el tema más habitual en las cuentas de Twitter de los cuatro principales líderes políticos españoles fueron las actividades y eventos de campaña protago- 
nizados por ellos mismo o, en general, por su propio partido. La excepción, relativa, durante esta campaña electoral fue Albert Rivera, quien dedicó proporcionalmente menos tuits a este asunto, lo que le permitió reforzar otros temas de interés para su estrategia electoral.

Puede afirmarse que, dejando a un lado el macrotema de las actividades de campaña, los asuntos que estuvieron presentes en las cuentas de los cuatro líderes y que, por lo tanto, pueden considerarse los issues de campaña compartidos en esta red social fueron los siguientes: la economía, la corrupción y la necesidad de regeneración, los derechos y servicios sociales, y la salida de Reino Unido de la Unión Europea, único tema este último de índole internacional.

Comparando entre líderes, se detectan diferencias entre ellos en relación con la mayor o menor importancia concedida a cada tema, lo que, junto con lo mostrado a continuación, demuestra la segunda hipótesis de este trabajo. En esta línea, los resultados permiten agrupar a los candidatos en función de la relevancia otorgada a determinados asuntos. De este modo, la economía y la organización territorial de España son dos asuntos priorizados por Rajoy y Rivera, lo que resulta coherente con el ideario del Partido Popular y de Ciudadanos en sus vertientes de centro-derecha y liberal. Iglesias y Sánchez, candidatos de los partidos de izquierda y centroizquierda, Podemos y Partido Socialista, tienen su principal nexo de unión temático en las reivindicaciones feministas y de género, asunto llamativamente inexistente en los tuits de Rajoy y de Rivera. La importancia concedida a los derechos y prestaciones sociales, incluyendo la educación y la sanidad públicas, entre otros, sirve para aglutinar los discursos de Sánchez, Iglesias y Rivera a través de un tema clásico en el discurso político de la izquierda que es también asumido por la vertiente centrista de Ciudadanos. Por último, hay un asunto que marca la línea divisoria entre viejos y nuevos partidos: la corrupción política y la necesidad de regeneración. El relieve que adquiere en las cuentas de los candidatos de Podemos y Ciudadanos contrasta con el menor que presenta en las de Sánchez y Rajoy, especialmente en la de este último. Una de las claves discursivas de los líderes emergentes es precisamente la de mostrar sus partidos como instrumentos de regeneración política frente a la corrupción que ha afectado fundamentalmente a las dos fuerzas representativas del bipartidismo.

\section{Referencias}

1. Abejón-Mendoza, P., Sastre-Asensio, A., y Linares-Rodríguez, V. (2012). Facebook y Twitter en campañas electorales en España. Anuario electrónico de estudios en comunicación social "Disertaciones", 5(1): 129-159.

2. Alonso-Muñoz, L., Marcos-García, S., y Casero-Ripollés, A. (2016). Political leaders in (inter)action. Twitter as a strategic communication tool in electoral campaigns. Trípodos, 39: 71-90. 
3. Cammaerts, B. (2012). Protest Logics and the Mediation Opportunity Structure. European Journal of Communication, 27(2): 117-134. doi:10.1177/0267323112441007.

4. Casero-Ripollés, A., Feenstra, R. A., y Tormey, S. (2016). Old and New Media Logics in an Electoral Campaign The Case of Podemos and the Two-Way Street Mediatization of Politics. The International Journal of Press/Politics, 21(3): 378-397. doi: 10.1177/1940161216645340.

5. Cea, M. A. (2001). Metodología cuantitativa. Estrategias y técnicas de investigación social. Madrid: Síntesis.

6. Cheong, P. H., y Lundry, C. (2012). Prosumption, Transmediation, and Resistance: Terrorism and Man-Hunting in Southeast Asia. American Behavioral Scientist, 56(4): 488-510. doi: 10.1177/0002764211429365.

7. Ellakuría, I., y De Paco, J. M. (2015). Alternativa naranja. Ciudadanos a la conquista de España. Barcelona: Editorial Debate.

8. García, C., y Zugasti, R. (2014). La campaña virtual en twitter: análisis de las cuentas de Rajoy y de Rubalcaba en las elecciones generales de 2011. Historia y Comunicación Social, 19(especial febrero): 299-311. doi:10.5209/rev_HICS.2014.v19.45029.

9. García, C., y Zugasti, R. (2016). Los temas de campaña en Twitter: caso de los candidatos a la presidencia de Aragón en 2015. Revista F@ro, 23(1): 181-194.

10. Jivkova-Semova, D., Requeijo-Rey, P., y Padilla-Castillo, G. (2017). Usos y tendencias de Twi er en la campaña a elecciones generales españolas del 20D de 2015: hashtags que fueron trending topic. El profesional de la información, 26(5): 824-837.

11. Lafuente, P., y Verón, J. J. (2013). El uso de Twitter por los líderes de las organizaciones políticas minoritarias en la campaña electoral de las generales de 2011. En: Crespo, I. (dir). Partidos, medios y electores en procesos de cambio. Valencia: Tirant: 541-562.

12. Lilleker, D. G., y Jackson, N. A. (2010). Towards a more participatory style of election campaigning: The impact of web 2.0 on the UK 2010 general election. Policy $\mathcal{E}$ internet, 2(3): 67-96. doi: 10.2202/1944-2866.1064.

13. López-García, G. (2016). 'Nuevos' y 'viejos' liderazgos: la campaña de las elecciones generales españolas de 2015 en Twitter. Comunicación y Sociedad, 29(3): 149-167.

14. Pallarés, S., y García, C. (2017). Análisis comparativo del discurso de Mariano Rajoy en Twitter durante las dos campañas electorales de 2015: las autonómicas del 24M y las generales del 20D. Revista Dígitos, 3(1): 119-136.

15. Rodríguez, R., y Ureña, D. (2012). Diez razones para el uso de Twitter como herramienta en la comunicación política y electoral. Comunicación y Pluralismo, 5: 89-116.

16. Santín, M., y Álvarez-Monzoncillo, J. M. (2017). El protagonismo de las redes sociales en la dieta informativa de los millenials. En Álvarez-Monzoncillo, J. M., y De Haro-Rodríguez, G. (Coords.). Millenials. La generación emprendedora. Madrid: Fundación Telefónica y Ariel: 79-108.

17. Zamora, R., y Zurutuza, C. (2014). Campaigning on Twitter: Towards the 'personal style' campaign to activate the political engagement during the 2011 Spanish general elections. Communication \& Society, 27(1): 83-106. 
18. Zamora-Medina, R., Sánchez-Cobarro, P. H., y Martínez-Martínez, H. (2017). The importance of the "strategic game" to frame the poliitcal discourse in Twitter during 2015 Spanish Regional Elections. Communication \& Society, 30(3): 229-253. doi: 10.15581/003.30.3.229-253.

19. Zugasti, R., y Sabés, F. (2015). Los issues de los candidatos en Twitter durante la campaña de las elecciones generales de 2011. ZER, 20(38): 161-178.

20. Zugasti, R., y Pérez, J. (2016). Los temas de campaña en Twitter de @PPopular y @ahorapodemos para las elecciones europeas de 2014. adComunica, 12: 205-223. doi:10.6035/2174-0992.2016.12.12

21. Zugasti, R., y García, C. (2018). Los temas de los líderes politico españoles en Twitter. Análisis de las dos campañas electorales de 2015. Icono 14, 16(1): 136-159. doi: 10.7195/ri14.v16i1.1137. 